\title{
DISCRETE POINCARÉ-TYPE INEQUALITIES
}

\author{
WING-SUM CHEUNG
}

\begin{abstract}
In this paper some discrete analogue of Poincaré-type integral inequalities involving many independent variables are established. These in turn can be used to serve as generators of other interesting discrete inequalities.
\end{abstract}

\section{Introduction}

It is well-recognized that integral inequalities in general provide a very useful and important device in the study of many qualitative as well as quantitative properties of solutions of differential equations. Among these are the so-called Poincaré-type integral inequalities which are, as indispensable tools as well as inspiring integral inequalities, of fundamental importance.

The original Poincaré's inequality is the multi-dimensional integral inequality $[6,10]$

$$
\lambda_{0} \int_{\Omega} f^{2} d x \leq \int_{\Omega}|\nabla f|^{2} d x
$$

where $\Omega$ is any bounded region in $\mathbb{R}^{2}$ or $\mathbb{R}^{3}, f \in C^{1}(\Omega), f=0$ on $\partial \Omega$, and $\lambda_{0}$ is the smallest eigenvalue of the problem

$$
\begin{cases}\triangle f+\lambda f=0 & \text { in } \Omega \\ f=0 & \text { on } \partial \Omega\end{cases}
$$

Because of its usefulness and importance, Poincaré's inequality has attracted much attention and a great deal of its generalizations to various aspects have been established in the literature. For instance, one can consult Beckenbach-Bellman [1], Hardy-LittlewoodPólya [5], Mitrinovic [10], Nirenberg [11], and more recently Horgan [6-8], Pachpatte [12, 13], Rassias [14, 15], Cheung [2-4] and Milovanovic-Mitrinovic-Rassias [9] for the details.

As can be anticipated, since the Poincaré-type integral inequalities are so important in the study of properties of solutions of differential equations, their discrete analogue should also be useful in the study of properties of solutions of difference equations. It is the purpose of this paper to give the discrete versions of some Poincaré-type inequalities given in $[3,4]$ by using methods that are rather elementary but yet inspiring in the sense

Received June 3, 1997.

1991 Mathematics Subject Classification. Primary 39A10, 39A12, 39B72.

Key words and phrases.Poincaré-type inequalities . 
that many other types of discrete inequalities could easily be arrived at by analogous methods.

\section{Notation and Preliminaries}

Throughout this paper, $m \geq 2$ and $n \geq 1$ will denote two fixed integers. Let $\alpha, \beta, \gamma, \ldots$ be indices running from 1 to $m$ and $i, j, k, \ldots$ from 1 to $n$. Let $\Omega=\prod_{i=1}^{n}\left[0, b_{i}\right] \cap \mathbb{Z}^{n} \subset \mathbb{R}^{n}$ be a fixed rectangular lattice of integral points, where for each $i, b_{i} \in \mathbb{N} \cup\{0\}$. A general point in $\Omega$ will be denoted by $t=\left(t_{1}, \ldots, t_{n}\right)$. The collection of real-valued functions on $\Omega$ and those which vanish on the boundary $\partial \Omega$ of $\Omega$ will be denoted by $\mathcal{F}(\Omega)$ and $\mathcal{F}_{0}(\Omega)$, respectively. For the sake of convenience, we shall extend the domain of definition of each function in $\mathcal{F}(\Omega)$, hence including those in $\mathcal{F}_{0}(\Omega)$, trivially to the entire $\mathbb{Z}^{n}$ and think of $\mathcal{F}(\Omega)$ as the collection of real-valued functions on $\mathbb{Z}^{n}$ with support in $\Omega$ and $\mathcal{F}_{0}(\Omega)$ as those with support in $\Omega \backslash \partial \Omega$.

For the sake of simplicity, summations and products over $\alpha, \beta, \gamma$ or $i, j, k$ will be abbreviated as $\sum_{\alpha}, \Pi_{i}$, etc., unless possible confusion may arise.

The following fundamental inequalities, which are easily obtainable from the quadratic mean-arithmetic mean-geometric mean inequality, will be needed in the sequal (see, e.g. $[5,10])$.

Lemma 1. For any $p_{\alpha}, q_{\alpha}, c_{\alpha}>0$ with $\sum q_{\alpha} / p_{\alpha}=1$,

$$
\prod_{\alpha} c_{\alpha}^{q_{\alpha}} \leq \sum_{\alpha} \frac{q_{\alpha}}{p_{\alpha}} c_{\alpha}^{p_{\alpha}}
$$

and the equality holds if and only if $c_{1}=\cdots=c_{m}$.

Lemma 2. For any $r_{i} \geq 0$ and $s \geq 0$,

$$
\left(\sum_{i} r_{i}\right)^{s} \leq c(s, n) \sum_{i} r_{i}^{s},
$$

where

$$
c(s, n)= \begin{cases}n^{s-1} & \text { if } s>1 \\ 1 & \text { if } 0 \leq s \leq 1\end{cases}
$$

\section{Main Results}

For any $f \in \mathcal{F}(\Omega)$, define

$$
f_{j}: \mathbb{Z}^{n} \rightarrow \mathbb{R}
$$

by

$$
\begin{aligned}
f_{j}\left(t_{1}, \ldots, t_{n}\right): & =\triangle_{j} f\left(t_{1}, \ldots, t_{n}\right) \\
& =f\left(t_{1}, \ldots, t_{j}, \ldots, t_{n}\right)-f\left(t_{1}, \ldots, t_{j}-1, \ldots, t_{n}\right) .
\end{aligned}
$$


Observe that if $f \in \mathcal{F}_{0}(\Omega), f_{j} \in \mathcal{F}(\Omega)$ for every $j$. However, in general $f_{j} \notin \mathcal{F}_{0}(\Omega)$. As usual, we define the gradient

$$
\nabla f:=\left(f_{1}, \ldots, f_{n}\right)
$$

and its norm

$$
|\nabla f|:=\left(\sum_{j}\left|f_{j}\right|^{2}\right)^{1 / 2}
$$

For any $p>0$, the $\ell_{p}$-norm of $f$ is deined as

$$
\|f\|_{p}:=\left(\sum_{t \in \Omega}|f(t)|^{p}\right)^{1 / p}, \quad f \in \mathcal{F}(\Omega)
$$

and the $\ell_{p}$-norm of $\nabla f$ as

$$
\|\nabla f\|_{p}:=\left(\sum_{t \in \Omega}|\nabla f(t)|^{p}\right)^{1 / p}=\left[\sum_{t \in \Omega}\left(\sum_{j}\left|f_{j}\right|^{2}\right)^{p / 2}\right]^{1 / p}, \quad f \in \mathcal{F}_{0}(\Omega) .
$$

Since $\Omega$ is finite, \|\|$_{p}$ exists for every $p>0$.

Let $B=\max \left\{b_{j}: j=1, \ldots, n\right\}$.

Theorem 1 . For any $f^{\alpha} \in \mathcal{F}_{0}(\Omega)$ and any real numbers $p_{\alpha} \geq 2$ with $\sum_{\alpha}\left(1 / p_{\alpha}\right)=$ 1 ,

$$
\left\|\prod_{\alpha} f^{\alpha}\right\|_{1} \leq \frac{1}{n} \sum_{\alpha} \frac{1}{p_{\alpha}}\left(\frac{B}{2}\right)^{p_{\alpha}}\left\|\nabla f^{\alpha}\right\|_{p_{\alpha}}^{p_{\alpha}}
$$

Theorem 2. For any $f^{\alpha} \in \mathcal{F}_{0}(\Omega)$ and any real numbers $q_{\alpha}>0$ with $q:=$ $\sum_{\alpha} q_{\alpha} \geq 2$

$$
\left\|\prod_{\alpha}\left(f^{\alpha}\right)^{q_{\alpha}}\right\|_{1} \leq \frac{1}{n}\left(\frac{B}{2}\right)^{q} \sum_{\alpha} \frac{q_{\alpha}}{q}\left\|\nabla f^{\alpha}\right\|_{q}^{q} .
$$

The following are some simple consequences of these theorems.

Corollary $\mathbb{1}$. For any $f^{\alpha} \in \mathcal{F}_{0}(\Omega)$,

$$
\left\|\prod_{\alpha} f^{\alpha}\right\|_{1} \leq \frac{1}{n m}\left(\frac{B}{2}\right)^{m} \sum_{\alpha}\left\|\nabla f^{\alpha}\right\|_{m}^{m}
$$

Proof. It follows easily from Theorem 1 by setting $p_{\alpha}=m$ for all $\alpha$ or from Theorem 2 by setting $q_{\alpha}=1$ for all $\alpha$.

Q.E.D.

Corollary 2. For any $f \in \mathcal{F}_{0}(\Omega)$

$$
\|f\|_{m}^{m} \leq \frac{1}{n}\left(\frac{B}{2}\right)^{m}\|\nabla f\|_{m}^{m}
$$


Proof. It follows immediately from Corollary 1 by letting $f^{\alpha}=f$ for all $\alpha$. Q.E.D.

Remark. Observe that in the inequality of Corollary 2, the number $m$ can be any integer $\geq 2$, not necessarily restricted to the fixed $m$ as mentioned before.

To prove Theorems 1 and 2, we first establish the following lemma.

Lemma 3. Let $f \in \mathcal{F}_{0}(\Omega)$. Then for any $t=\left(t_{1}, \ldots, t_{n}\right) \in \Omega$,

$$
|f(t)| \leq \frac{1}{2 n} \sum_{j} \sum_{u_{j}=1}^{b_{j}}\left|f_{j}\left(t_{1}, \ldots, t_{j-1}, u_{j}, t_{j+1}, \ldots, t_{n}\right)\right| .
$$

Proof. Since $f$ vanishes on $\partial \Omega$, for each $j=1, \ldots, n$ we have

$$
f(t)=\sum_{u_{j}=1}^{t_{j}} f_{j}\left(t_{1}, \ldots, t_{j-1}, u_{j}, t_{j+1}, \ldots, t_{n}\right)
$$

and also

$$
f(t)=-\sum_{u_{j}=t_{j}+1}^{b_{j}} f_{j}\left(t_{1}, \ldots, t_{j-1}, u_{j}, t_{j+1}, \ldots, t_{n}\right) .
$$

Taking their absolute values and summing up with respect to $j$, we have

$$
2 n|f(t)| \leq \sum_{j} \sum_{u_{j}=1}^{b}\left|f_{j}\left(t_{1}, \ldots, t_{j-1}, u_{j}, t_{j+1}, \ldots, t_{n}\right)\right|
$$

hence the lemma.

Proof of Theorem 1 . By Lemma 1 with $q_{\alpha}=1$, Lemma 3, and Lemma 2, we have

$$
\begin{aligned}
\prod_{\alpha}\left|f^{\alpha}(t)\right| & \leq \sum_{\alpha} \frac{1}{p_{\alpha}}\left|f^{\alpha}(t)\right|^{p_{\alpha}} \\
& \leq \sum_{\alpha} \frac{1}{p_{\alpha}}\left[\frac{1}{2 n} \sum_{j} \sum_{u_{j}=1}^{b_{j}}\left|f_{j}^{\alpha}\left(t_{1}, \ldots, t_{j-1}, u_{j}, t_{j+1}, \ldots, t_{n}\right)\right|\right]^{p_{\alpha}} \\
& \leq \sum_{\alpha} \frac{1}{p_{\alpha}}\left(\frac{1}{2 n}\right)^{p_{\alpha}}\left[c\left(p_{\alpha}, n\right) \sum_{j}\left(\sum_{u_{j}=1}^{b_{j}}\left|f_{j}^{\alpha}\left(t_{1}, \ldots, t_{j-1}, u_{j}, t_{j+1}, \ldots, t_{n}\right)\right|\right)^{p_{\alpha}}\right] .
\end{aligned}
$$

Since $p_{\alpha} \geq 2>1$, we have $c\left(p_{\alpha}, n\right)=n^{p_{\alpha}-1}$ for all $\alpha$ and so by Hölder's inequality,

$$
\begin{aligned}
\prod_{\alpha}\left|f^{\alpha}(t)\right| & \leq \sum_{\alpha} \frac{1}{n p_{\alpha}}\left(\frac{1}{2}\right)^{p_{\alpha}} \sum_{j}\left(\sum_{u_{j}=1}^{b_{j}}\left|f_{j}^{\alpha}\left(t_{1}, \ldots, t_{j-1}, u_{j}, t_{j+1}, \ldots, t_{n}\right)\right|\right)^{p_{\alpha}} \\
& \leq \sum_{\alpha} \frac{1}{n p_{\alpha}}\left(\frac{1}{2}\right)^{p_{\alpha}} \sum_{j}\left[\left(\sum_{u_{j}=1}^{b_{j}}\left|f_{j}^{\alpha}\left(t_{1}, \ldots, t_{j-1}, u_{j}, t_{j+1}, \ldots, t_{n}\right)\right|^{p_{\alpha}}\right)^{1 / p_{\alpha}}\right.
\end{aligned}
$$




$$
\begin{aligned}
& \left.\cdot\left(\sum_{u_{j}=1}^{b_{j}} 1\right)^{\frac{p_{\alpha}-1}{p_{\alpha}}}\right]^{p_{\alpha}} \\
= & \sum_{\alpha} \frac{1}{n p_{\alpha}}\left(\frac{1}{2}\right)^{p_{\alpha}} \sum_{j}\left[b_{j}^{p_{\alpha}-1} \sum_{u_{j}=1}^{b_{j}}\left|f_{j}^{\alpha}\left(t_{1}, \ldots, t_{j-1}, u_{j}, t_{j+1}, \ldots, t_{n}\right)\right|^{p_{\alpha}}\right] \\
\leq & \sum_{\alpha} \frac{1}{n p_{\alpha}}\left(\frac{1}{2}\right)^{p_{\alpha}-1} B^{p_{\alpha}-1} \sum_{j} \sum_{u_{j}=1}^{b_{j}}\left|f_{j}^{\alpha}\left(t_{1}, \ldots, t_{j-1}, u_{j}, t_{j+1}, \ldots, t_{n}\right)\right|^{p_{\alpha}} .
\end{aligned}
$$

Therefore,

$$
\begin{aligned}
& \sum_{t \in \Omega}\left|\prod_{\alpha} f^{\alpha}(t)\right| \\
\leq & \sum_{t \in \Omega}\left[\sum_{\alpha} \frac{1}{n p_{\alpha}}\left(\frac{1}{2}\right)^{p_{\alpha}} B^{p_{\alpha}-1} \sum_{j} \sum_{u_{j}=1}^{b_{j}}\left|f_{j}^{\alpha}\left(t_{1}, \ldots, t_{j-1}, u_{j}, t_{j+1}, \ldots, t_{n}\right)\right|^{p_{\alpha}}\right] \\
= & \sum_{\alpha} \frac{1}{n p_{\alpha}}\left(\frac{1}{2}\right)^{p_{\alpha}} B^{p_{\alpha}-1} \sum_{j}\left[\sum_{t \in \Omega} \sum_{u_{j}=1}^{b_{j}}\left|f_{j}^{\alpha}\left(t_{1}, \ldots, t_{j-1}, u_{j}, t_{j+1}, \ldots, t_{n}\right)\right|^{p_{\alpha}}\right] \\
= & \sum_{\alpha} \frac{1}{n p_{\alpha}}\left(\frac{1}{2}\right)^{p_{\alpha}} B^{p_{\alpha}-1} \sum_{j}\left[\sum_{u_{j}=1}^{b_{j}} \sum_{t \in \Omega}\left|f_{j}^{\alpha}\left(t_{1}, \ldots, t_{j-1}, t_{j}, t_{j+1}, \ldots, t_{n}\right)\right|^{p_{\alpha}}\right] \\
= & \sum_{\alpha} \frac{1}{n p_{\alpha}}\left(\frac{1}{2}\right)^{p_{\alpha}} B^{p_{\alpha}-1} \sum_{j}\left[b_{j} \sum_{t \in \Omega}\left|f_{j}^{\alpha}(t)\right|^{p_{\alpha}}\right] \\
\leq & \sum_{\alpha} \frac{1}{n p_{\alpha}}\left(\frac{B}{2}\right)^{p_{\alpha}} \sum_{j} \sum_{t \in \Omega}\left|f_{j}^{\alpha}(t)\right|^{p_{\alpha}} \\
= & \sum_{\alpha} \frac{1}{n p_{\alpha}}\left(\frac{B}{2}\right)^{p_{\alpha}} \sum_{t \in \Omega}\left(\sum_{j}\left|f_{j}^{\alpha}(t)\right|^{p_{\alpha}}\right) \\
\leq & \sum_{\alpha} \frac{1}{n p_{\alpha}}\left(\frac{B}{2}\right)^{p_{\alpha}} \sum_{t \in \Omega}\left[\left(\sum_{j}\left|f_{j}^{\alpha}(t)\right|^{p_{\alpha}}\right)^{2 / p_{\alpha}}\right]^{p_{\alpha} / 2} \\
\leq & \sum_{\alpha} \frac{1}{n p_{\alpha}}\left(\frac{B}{2}\right)^{p_{\alpha}} \sum_{t \in \Omega}\left[c\left(\frac{2}{p_{\alpha}}, n\right) \sum_{j}\left(\left|f_{j}^{\alpha}(t)\right|^{p_{\alpha}}\right)^{2 / p_{\alpha}}\right]^{p_{\alpha} / 2}
\end{aligned}
$$

by Lemma 2 . Since $p_{\alpha} \geq 2$, we have $c\left(\frac{2}{p_{\alpha}}, n\right)=1$ for all $\alpha$ and so

$$
\begin{aligned}
\sum_{t \in \Omega}\left|\prod_{\alpha} f^{\alpha}(t)\right| & \leq \sum_{\alpha} \frac{1}{n p_{\alpha}}\left(\frac{B}{2}\right)^{p_{\alpha}} \sum_{t \in \Omega}\left(\sum_{j}\left|f_{j}^{\alpha}(t)\right|^{2}\right)^{p_{\alpha} / 2} \\
& =\frac{1}{n} \sum_{\alpha} \frac{1}{p_{\alpha}}\left(\frac{B}{2}\right)^{p_{\alpha}} \sum_{t \in \Omega}\left|\nabla f^{\alpha}(t)\right|^{p_{\alpha}}
\end{aligned}
$$

hence the theorem. 
Proof of Theorem 2. By Lemma 1 with $p_{\alpha}=q$ for all $\alpha$, Lemma 3, and Lemma 2 , we have

$$
\begin{aligned}
\prod_{\alpha}\left|f^{\alpha}(t)\right|^{q_{\alpha}} & \leq \frac{1}{q} \sum_{\alpha} q_{\alpha}\left|f^{\alpha}(t)\right|^{q} \\
& \leq \frac{1}{q} \sum_{\alpha} q_{\alpha}\left[\frac{1}{2 n} \sum_{j} \sum_{u_{j}=1}^{b_{j}}\left|f_{j}^{\alpha}\left(t_{1}, \ldots, t_{j-1}, u_{j}, t_{j+1}, \ldots, t_{n}\right)\right|\right]^{q} \\
& \leq \frac{1}{q} \sum_{\alpha} q_{\alpha}\left(\frac{1}{2 n}\right)^{q}\left[c(q, n) \sum_{j}\left(\sum_{u_{j}=1}^{b_{j}}\left|f_{j}^{\alpha}\left(t_{1}, \ldots, t_{j-1}, u_{j}, t_{j+1}, \ldots, t_{n}\right)\right|\right)^{q}\right] .
\end{aligned}
$$

Since $q=\sum q_{\alpha} \geq 2$, we have $c(q, n)=n^{q-1}$ and so by Hölder's inequality,

$$
\begin{aligned}
\prod_{\alpha}\left|f^{\alpha}(t)\right|^{q_{\alpha}} \leq & \frac{1}{n q}\left(\frac{1}{2}\right)^{q} \sum_{\alpha, j} q_{\alpha}\left(\sum_{u_{j}=1}^{b_{j}}\left|f_{j}^{\alpha}\left(t_{1}, \ldots, t_{j-1}, u_{j}, t_{j+1}, \ldots, t_{n}\right)\right|\right)^{q} \\
\leq & \frac{1}{n q}\left(\frac{1}{2}\right)^{q} \sum_{\alpha, j} q_{\alpha}\left[\left(\sum_{u_{j}=1}^{b_{j}}\left|f_{j}^{\alpha}\left(t_{1}, \ldots, t_{j-1}, u_{j}, t_{j+1}, \ldots, t_{n}\right)\right|^{q}\right)^{\frac{1}{q}}\right. \\
& \left.\cdot\left(\sum_{u_{j}=1}^{b_{j}} 1\right)^{\frac{q-1}{q}}\right]^{q} \\
= & \frac{1}{n q}\left(\frac{1}{2}\right)^{q} \sum_{\alpha, j} q_{\alpha}\left[b_{j}^{q-1} \sum_{u_{j}=1}^{b_{j}}\left(\left.f_{j}^{\alpha}\left(t_{1}, \ldots, t_{j-1}, u_{j}, t_{j+1}, \ldots, t_{n}\right)\right|^{q}\right]\right. \\
\leq & \frac{1}{n q}\left(\frac{1}{2}\right)^{q} B^{q-1} \sum_{\alpha, j} q_{\alpha}\left[\sum_{u_{j}=1}^{b_{j}}\left|f_{j}^{\alpha}\left(t_{1}, \ldots, t_{j-1}, u_{j}, t_{j+1}, \ldots, t_{n}\right)\right|^{q}\right] .
\end{aligned}
$$

Therefore

$$
\begin{aligned}
& \sum_{t \in \Omega}\left|\prod_{\alpha}\left(f^{\alpha}(t)\right)^{q_{\alpha}}\right| \\
\leq & \sum_{i \in \Omega}\left[\frac{1}{n q}\left(\frac{1}{2}\right)^{q} B^{q-1} \sum_{\alpha, j} q_{\alpha}\left(\sum_{u_{j}=1}^{b_{j}}\left|f_{j}^{\alpha}\left(t_{1}, \ldots, t_{j-1}, u_{j}, t_{j+1}, \ldots, t_{n}\right)\right|^{q}\right)\right] \\
= & \frac{1}{n q}\left(\frac{1}{2}\right)^{q} B^{q-1} \sum_{\alpha, j} q_{\alpha} \sum_{t \in \Omega} \sum_{u_{j}=1}^{b_{j}}\left|f_{j}^{\alpha}\left(t_{1}, \ldots, t_{j-1}, u_{j}, t_{j+1}, \ldots, t_{n}\right)\right|^{q} \\
= & \frac{1}{n q}\left(\frac{1}{2}\right)^{q} B^{q-1} \sum_{\alpha, j} q_{\alpha} \sum_{u_{j}=1} \sum_{t \in \Omega}\left|f_{j}^{\alpha}\left(t_{1}, \ldots, t_{j-1}, t_{j}, t_{j+1}, \ldots, t_{n}\right)\right|^{q} \\
= & \frac{1}{n q}\left(\frac{1}{2}\right)^{q} B^{q-1} \sum_{\alpha, j} q_{\alpha} \cdot b_{j} \cdot\left(\sum_{t \in \Omega}\left|f_{j}^{\alpha}(t)\right|^{q}\right)
\end{aligned}
$$




$$
\begin{aligned}
& \leq \frac{1}{n q}\left(\frac{B}{2}\right)^{q} \sum_{\alpha} q_{\alpha}\left[\sum_{j}\left(\sum_{t \in \Omega}\left|f_{j}^{\alpha}(t)\right|^{q}\right)\right] \\
& \left.=\frac{1}{n q}\left(\frac{B}{2}\right)^{q} \sum_{\alpha} q_{\alpha} \sum_{t \in \Omega}\left(\sum_{j}\left|f_{j}^{\alpha}(t)\right|^{q}\right)\right] \\
& =\frac{1}{n q}\left(\frac{B}{2}\right)^{q} \sum_{\alpha} q_{\alpha} \sum_{t \in \Omega}\left[\left(\sum_{j}\left|f_{j}^{\alpha}(t)\right|^{q}\right)^{2 / q}\right]^{q / 2} \\
& \leq \frac{1}{n q}\left(\frac{B}{2}\right)^{q} \sum_{\alpha} q_{\alpha} \sum_{t \in \Omega}\left[c\left(\frac{2}{q}, n\right) \sum_{j}\left(\left|f_{j}^{\alpha}(t)\right|^{q}\right)^{2 / q}\right]^{q / 2}
\end{aligned}
$$

by Lemma 2 . Since $\frac{2}{q} \leq 1, c\left(\frac{2}{q}, n\right)=1$ and so

$$
\begin{aligned}
\sum_{t \in \Omega}\left|\prod_{\alpha}\left(f^{\alpha}(t)\right)^{q_{\alpha}}\right| & \leq \frac{1}{n q}\left(\frac{B}{2}\right)^{q} \sum_{\alpha} q_{\alpha} \sum_{t \in \Omega}\left(\sum_{j}\left|f_{j}^{\alpha}(t)\right|^{2}\right)^{q / 2} \\
& =\frac{1}{n}\left(\frac{B}{2}\right)^{q} \sum_{\alpha} \frac{q_{\alpha}}{q} \sum_{t \in \Omega}\left|\nabla f^{\alpha}(t)\right|^{q} .
\end{aligned}
$$

Hence the theorem follows.

Corollary 3. For any $f^{\alpha} \in \mathcal{F}_{0}(\Omega)$ and any real numbers $p_{\alpha} \geq 2$ with $\sum_{\alpha}\left(1 / p_{\alpha}\right)=1$,

$$
\left\|\sum_{\beta}\left(\prod_{\alpha \neq \beta} f^{\alpha}\right)\left|\nabla f^{\beta}\right|\right\|_{1} \leq\left(\frac{B}{2}\right)^{m-1}\left(\sum_{\alpha}\left(\frac{1}{n}\right)^{1-\frac{1}{p_{\alpha}}}\right)\left(\sum_{\alpha} \frac{1}{p_{\alpha}}\left\|\nabla f^{\alpha}\right\|_{p_{\alpha}}^{p_{\alpha}}\right) .
$$

Proof. By a generalization of Hölder's inequality and Corollary 2, we have

$$
\begin{aligned}
& \sum_{t \in \Omega}\left[\sum_{\beta}\left(\prod_{\alpha \neq \beta}\left|f^{\alpha}(t)\right|\right)\left|\nabla f^{\beta}(t)\right|\right] \\
& =\sum_{\beta} \sum_{t \in \Omega}\left[\left(\prod_{\alpha \neq \beta}\left|f^{\alpha}(t)\right|\right)\left|\nabla f^{\beta}(t)\right|\right] \\
& \left.\leq \sum_{\beta}\left[\prod_{\alpha \neq \beta}\left(\sum_{t \in \Omega}\left|f^{\alpha}(t)\right|^{p_{\alpha}}\right)^{1 / p_{\alpha}}\right]\left[\sum_{t \in \Omega}\left|\nabla f^{\beta}(t)\right|^{p_{\beta}}\right)^{1 / p_{\beta}}\right] \\
& \leq \sum_{\beta}\left[\prod_{\alpha \neq \beta}\left(\frac{1}{n}\left(\frac{B}{2}\right)^{p_{\alpha}}\left\|\nabla f^{\alpha}\right\|_{p_{\alpha}}^{p_{\alpha}}\right)^{1 / p_{\alpha}}\right]\left[\left(\left\|\nabla f^{\beta}\right\|_{p_{\beta}}^{p_{\beta}}\right)^{1 / p_{\beta}}\right] \\
& =\sum_{\beta}\left(\frac{1}{n}\right)^{\sum_{\alpha \neq \beta} \frac{1}{p_{\alpha}}}\left(\frac{B}{2}\right)^{\sum_{\alpha \neq \beta} 1} \cdot \prod_{\alpha}\left(\left\|\nabla f^{\alpha}\right\|_{p_{\alpha}}\right) \\
& =\left(\sum_{\beta}\left(\frac{1}{n}\right)^{1-\frac{1}{p_{\beta}}}\left(\frac{B}{2}\right)^{m-1}\right) \prod_{\alpha}\left(\left\|\nabla f^{\alpha}\right\|_{p_{\alpha}}\right) \text {. }
\end{aligned}
$$


Now by Lemma 1 it is not hard to see that

$$
\begin{aligned}
\prod_{\alpha}\left(\left\|\nabla f^{\alpha}\right\|_{p_{\alpha}}\right) & =\prod_{\alpha}\left(\sum_{t \in \Omega}\left|\nabla f^{\alpha}(t)\right|^{p_{\alpha}}\right)^{1 / p_{\alpha}} \\
& \leq \sum_{\alpha} \frac{1 / p_{\alpha}}{\left(\sum_{\gamma} 1 / p_{\gamma}\right)}\left(\sum_{t \in \Omega}\left|\nabla f^{\alpha}(t)\right|^{p_{\alpha}}\right)^{\sum_{\gamma} 1 / p_{\gamma}} \\
& =\sum_{\alpha} \frac{1}{p_{\alpha}}\left(\sum_{t \in \Omega}\left|\nabla f^{\alpha}(t)\right|^{p_{\alpha}}\right) \\
& =\sum_{\alpha} \frac{1}{p_{\alpha}}\left\|\nabla f^{\alpha}\right\|_{p_{\alpha}}^{p_{\alpha}}
\end{aligned}
$$

therefore

$$
\begin{aligned}
& \sum_{t \in \Omega}\left[\sum_{\beta}\left(\prod_{\alpha \neq \beta}\left|f^{\alpha}(t)\right|\right)\left|\nabla f^{\beta}(t)\right|\right] \\
\leq & \left(\sum_{\beta}\left(\frac{1}{n}\right)^{1-\frac{1}{p_{\beta}}}\left(\frac{B}{2}\right)^{m-1}\right) \sum_{\alpha} \frac{1}{p_{\alpha}}\left\|\nabla f^{\alpha}\right\|_{p_{\alpha}}^{p_{\alpha}} \\
= & \left(\frac{B}{2}\right)^{m-1}\left(\sum_{\alpha}\left(\frac{1}{n}\right)^{1-\frac{1}{p_{\alpha}}}\right)\left(\sum_{\alpha} \frac{1}{p_{\alpha}}\left\|\nabla f^{\alpha}\right\|_{p_{\alpha}}^{p_{\alpha}}\right) .
\end{aligned}
$$

Corollary 4. For any $f^{\alpha} \in \mathcal{F}_{0}(\Omega)$,

$$
\left\|\sum_{\beta}\left(\prod_{\alpha \neq \beta} f^{\alpha}\right)\left|\nabla f^{\beta}\right|\right\|_{1} \leq\left(\frac{B}{2}\right)^{m-1}\left(\frac{1}{n}\right)^{1-\frac{1}{m}} \sum_{\alpha}\left\|\nabla f^{\alpha}\right\|_{m}^{m} .
$$

Proof. It follows immediately from Corollary 3 by letting $p_{\alpha}=m$ for all $\alpha$.

Remark. Further interesting discrete inequalities of the Poincaré type can easily be generated from the results above. For instance, by taking $m=2$ in Corollary 4 , we get

$$
\|f|\nabla g|+g \mid \nabla f\|_{1} \leq \frac{B}{2 \sqrt{n}}\left(\|\nabla f\|_{2}^{2}+\|\nabla g\|_{2}^{2}\right)
$$

and by putting $f=g$ in the last inequality, we obtain

$$
\|f \mid \nabla f\|_{1} \leq \frac{B}{2 \sqrt{n}}\|\nabla f\|_{2}^{2} .
$$

These discrete inequalities are of great interest and useful in the study of qualitative as well as quantitative properties of solutions of difference equations. 


\section{References}

[1] E. F. Beckenbach and R. Bellman, Inequalities, Springer-Verlag, New York, 1965.

[2] W. S. Cheung, "On integral inequalities of the Sobolev type," Aequationes Math. 49(1995), 153-159.

[3] .W. S. Cheung, "On Poincaré type integral inequalities," Proc. Amer. Math. Soc. 119, No.3 (1993), 857-863.

[4] W. S. Cheung, "Some multi-dimensional integral inequalities," to appear.

[5] G. H. Hardy, J. E. Littlewood and G. Pólya, Inequalities, Cambridge Univ. Press, Cambridge, 1952.

[6] C. O. Horgan, "Integral bounds for solutions of nonlinear reaction-diffusion equations," $J$. Appl. Math. Phys. (ZAMP) 28(1977), 197-204.

[7] C. O. Horgan and R. R. Nachlinger, "On the domain of attraction for steady states in heat conduction," Internat. J. Engrg. Sci. 14(1976), 143-148.

[8] C. O. Horgan and L. T. Wheeler, "Spatial decay estimates for the Navier-Stokes equations with applications to the problem of entry flow," SIAM J. Appl. Math. 35(1978), 97-116.

[9] G. V. Milovanović, D. S. Mitrinović and Th. M. Rassias, Topics in Polynomials: Extremal Problems, Inequalities, Zeros, World Scientific Publ. Co., 1994.

[10] D. S. Mitrinović, Analytic inequalities, Springer-Verlag, Berlin, 1970.

[11] L. Nirenberg, "On elliptic partial differential equations," Ann. Scuola Norm. Sup. Pisa Cl. Sci. (4)13(1959), 116-162.

[12] B. G. Pachpatte, "On Poincaré-type integral inequalities," J. Math. Anal. Appl. 114(1986), 111-115.

[13] B. G. Pachpatte, "On some new integral inequalities in several independent variables," Chinese J. Math. 14(1986), 69-79.

[14] Th. M. Rassias, "On certain properties of eigenvalues and the Poincaré inequality", Global Analysis-Analysis on Manifolds, Teubner-Texte zur Math, Teubner Leipzig, Band 57(1983), 282-300.

[15] Th. M. Rassias, "Un contre-exemple á l'inégalité de Poincaré," C. R. Acad. Sciences Paris $284(1977), 409-412$.

Department of Mathematics, University of Hong Kong, Pokfulam Road, Hong Kong. 